\title{
Probing anomalous off-shell electroweak dipole moment of the top quark
}

\author{
Edward Boos ${ }^{1,2}$, Vyacheslav Bunichev ${ }^{1}$, Lev Dudko ${ }^{1}$, and Georgii Vorotnikov ${ }^{1}$ \\ ${ }^{1}$ Skobeltsyn Institute of Nuclear Physics M.V. Lomonosov Moscow State University, Moscow 119991, Rus- \\ sian Federation \\ ${ }^{2}$ Faculty of Physics M.V.Lomonosov Moscow State University Leninskie Gory, Moscow 119991, Russian \\ Federation
}

\begin{abstract}
A possibility to search for anomalous Wtb interaction $\partial^{\mu} W_{\mu}^{-} \bar{b}\left(f_{\mathrm{W}}^{\mathrm{L}} P_{\mathrm{L}}+f_{\mathrm{W}}^{\mathrm{R}} P_{\mathrm{R}}\right) t$ is considered for the first time. Despite such couplings do not contribute to both pair top quark production or three main electroweak production modes, they could provide non-zero contributions to associated single top quark production with $\mathrm{W}$ or $\mathrm{Z}$ bosons or in association with two forward light jets via electroweak fusion diagrams.
\end{abstract}

\section{Introduction}

The Standard Model (SM) is a theory, which is now tested and proved in a hundreds of different experiments. However, SM obliviously is not a final theory, but a very successful effective theory within the electroweak energy scale. One of the primary goal of the Large Hadron Collider is to search for physics beyond the Standard Model (BSM). A new physics may manifest itself through different ways. Many BSM theories predict new particles if the energy of collision is greater than the production threshold ( $\left.E_{\text {collision }}>E_{\text {threshold }}\right)$. However, even in opposite scenario, when $\left(E_{\text {collision }}<E_{\text {threshold }}\right)$ BSM physics could prove itself through anomalous couplings originating from higher dimensional gauge invariant operators or interference terms of new resonances with the SM particles, that leads to deviation from SM prediction in production cross sections and/or kinematic distributions. The top quark sector of SM owing to its unique properties such as large mass, small lifetime, spin correlations and very small mixing to the light quark generations, could be a window to BSM physics. The effective Lagrangian approach is common used in experimental physics for model-independent description possible effects of BSM physics. Thus, lowest-dimension Lagrangian [1] for the Wtb vertex usually written as:

$$
\mathfrak{L}=\frac{g}{\sqrt{2}} \overline{\mathrm{b}} \gamma^{\mu}\left(f_{\mathrm{V}}^{\mathrm{L}} P_{\mathrm{L}}+f_{\mathrm{V}}^{\mathrm{R}} P_{\mathrm{R}}\right) \mathrm{t} \mathrm{W}_{\mu}^{-}-\frac{g}{\sqrt{2}} \overline{\mathrm{b}} \frac{\sigma^{\mu v} \partial_{\nu} \mathrm{W}_{\mu}^{-}}{M_{\mathrm{W}}}\left(f_{\mathrm{T}}^{\mathrm{L}} P_{\mathrm{L}}+f_{\mathrm{T}}^{\mathrm{R}} P_{\mathrm{R}}\right) \mathrm{t}+\text { h.c. }
$$

where $P_{\mathrm{L}, \mathrm{R}}=\left(1 \mp \gamma_{5}\right) / 2, \sigma_{\mu \nu}=i\left(\gamma_{\mu} \gamma_{\nu}-\gamma_{\nu} \gamma_{\mu}\right) / 2, g$ is the coupling constant of the weak interaction, the constant $f_{\mathrm{V}}^{\mathrm{L}}$ represents the left-handed vector coupling, presented in $\mathrm{SM}, f_{\mathrm{V}}^{\mathrm{R}}$ is the anomalous right-handed vector coupling and $f_{\mathrm{T}}^{\mathrm{L}}\left(f_{\mathrm{T}}^{\mathrm{R}}\right)$ represents the anomalous left-handed (right-handed) tensor coupling. Such anomalous couplings nowadays are relatively well studied both theoretically [2]-[5] and experimentally [6]-[8]. In this paper we discuss additional anomalous couplings which were not considered yet from phenomenology point of view. 


\section{Relevant processes}

As was mentioned in the paper [1] there is an additional contribution to the effective Lagrangian in case of off-shell W boson:

$$
\partial^{\mu} W_{\mu}^{-} \bar{b} P_{\mathrm{L}} t
$$

Such an interaction which one can call "off-shell electroweak dipole moment" (osEDM) appears in the effective electroweak non-linear chiral Lagrangian approach [9]. Phenomenological study of this interaction term has significant difficulties. The interaction term is totally vanish for an on-shell W boson or in the case $\mathrm{W}$ couples to massless on-shell fermions. Therefore, the anomalous osEDM couplings do not contribute to the main top quark production processes, both pair or any of three electroweak production t-, s-, tW-channels.

Despite such couplings do not contribute to usual top quark production processes they could provide non-zero contributions to associated single top quark production with $\mathrm{W}$ or $\mathrm{Z}$ bosons or in association with two forward light jets via electroweak fusion diagrams. The representative Feynman diagrams with non-zero osEDM contribution are shown in Fig. 1. In this paper we consider more simple $2 \rightarrow 3$ processes of associated production top quark and electroweak gauge bosons.
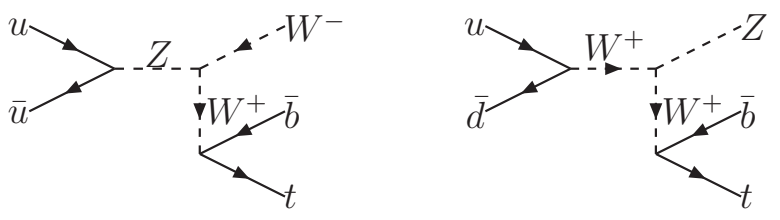

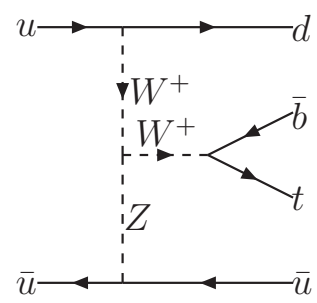

Figure 1. Feynman diagrams of the processes, which could be used to search for the anomalous off-shell W boson couplings.

The effective Lagrangian used in the analysis has the following form:

$$
\mathfrak{L}_{e f f .}=\frac{g}{\sqrt{2}} \frac{v}{\Lambda^{2}} \partial^{\mu} W_{\mu}^{-} \bar{b}\left(f_{\mathrm{W}}^{\mathrm{L}} P_{\mathrm{L}}+f_{\mathrm{W}}^{\mathrm{R}} P_{\mathrm{R}}\right) t+\text { h.c.. }
$$

Here $\frac{v}{\Lambda^{2}}$ corresponds to natural dimensional factor of the anomalous operators, $v=246 \mathrm{GeV}$ is vacuum expectation value, $\Lambda$ is the scale of a new physics and constants $f_{\mathrm{W}}^{\mathrm{L}}$ and $f_{\mathrm{W}}^{\mathrm{R}}$ are the strengths of left and right anomalous couplings. In the purpose of CP conserving, we consider the case of a purely imaginary $f_{\mathrm{W}}^{\mathrm{L}}$ and $f_{\mathrm{W}}^{\mathrm{R}}$ values [9].

For studying processes with anomalous osEDM the CompHEP 4.5.2 package [10] was used to model kinematic distributions of the osEDM and SM contributions including their interference. The Feynman rules for the Wtb vertex were generated with the LanHEP package [11], and implemented into CompHEP.

The complete set of electroweak diagrams for the associated tbZ production is shown in Fig. 2. The first three diagrams give non-zero contribution with considered anomalous coupling while the last two contain only SM contributions. The second process tbW also contains non-zero osEDM contributions from the diagrams like the first diagram in Fig 1. However, in this case there are SM diagrams corresponding to top quark pair production and associated tW single top production with 
cross sections significantly larger than possible anomalous contributions. Moreover, in case of tWb final state there is contribution from gluon - gluon collision with huge SM rate and zero osEDM contribution.

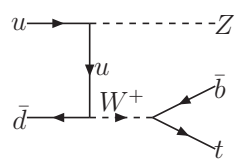

a)

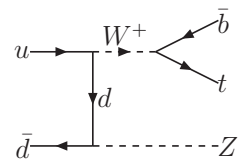

b)

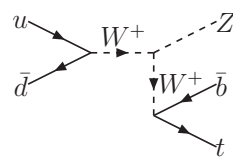

c)

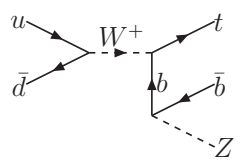

d)

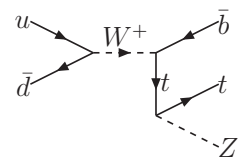

e)

Figure 2. All relevant Feynman diagrams of the process of associated production of a top quark with $\mathrm{Z}$ boson.

\section{Kinematic distribution and extracting potential}

Since anomalous osEDM interactions are directly proportional to the momentum of the W boson, it gives approximately linear grow of sensitivity to the couplings with the energy of the colliding protons. Thus, for such projects as High Energy LHC or Future Circular Collider, efficiency of the searches for osEDM couplings will be increasing 5-10 times compare to current energies at the LHC.

The anomalous interactions could manifest itself not only in cross section rates, but also in different kinematics of the process. For example, on Fig. 3 one can see distribution of a transverse momentum of the $\mathrm{Z}$ boson in the process of its associated production with top quark. In the case of anomalous contributions, the momentum, in average, is much higher than for SM production, and is peaking at about $160 \mathrm{GeV}$ instead of $\approx 50 \mathrm{GeV}$ for $\mathrm{SM}$. The same situation is observed for the transverse momenta of the $t$ and $b$ quarks.

The difference in transverse momentum distributions of the final state particles is provided mostly by their space bearings, as one can see on the Fig. 4. The asymmetry between the negative and positive parts of the plots is provided by choose of initial state quarks (ud), which gives symmetric picture with a complementary $\overline{\mathrm{d} u}$ initial state.

The angular distributions (Fig. 5) are also shown different behaviour of SM and osEDM production. The anomalous interactions are characterized by more smooth distribution both for $\mathrm{Zt}$ or tb angle.

However, one could see there are pure left and right osEDM interactions are totally indistinguishable on the Fig. 3-5. The way to see the difference between the couplings is in their interference with the SM part. The right anomalous interaction has a negative interference term with SM, while the left coupling has a small, but positive one (Fig. 6). Since there are no experimental limits on osEDM couplings we take such values for the couplings to get visible deviations from SM.

Another distinction between the coupling is the cross section rate for sea massive quarks in the initial state (Fig. 7). The difference is provided by diagrams a) and b) on the Fig 2, where quarks of different flavours are taken into account for propagators of the initial virtual quark exchange.

\section{Conclusion}

The possible anomalous osEDM interactions are phenomenologically studied for the first time. Such couplings do not contribute to both pair top quark production or three main electroweak production processes. However, there are several processes considered in this article which have non zero 

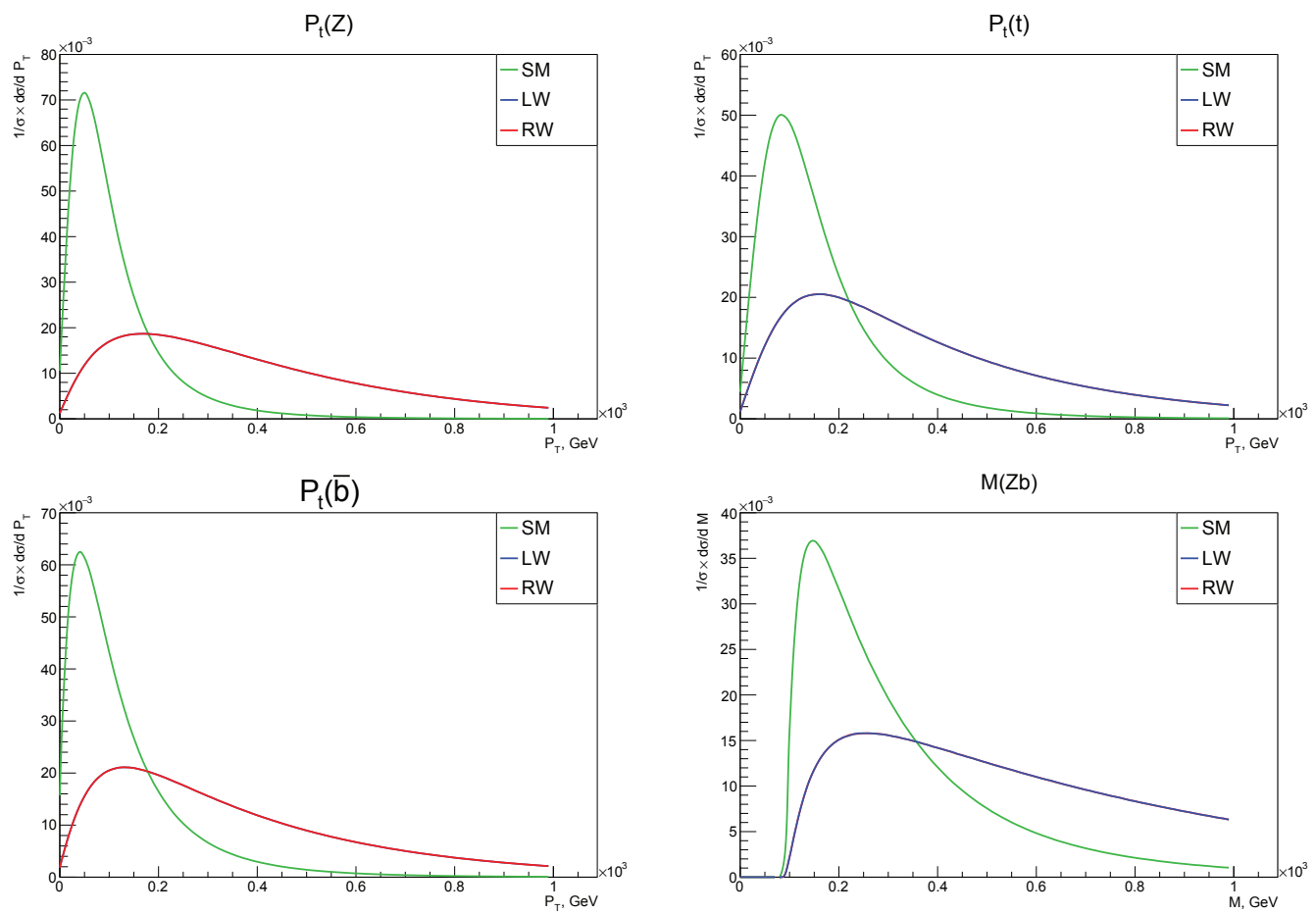

Figure 3. Transverse momentum distributions for the SM interaction (SM) and pure left (LW) and right (RW)

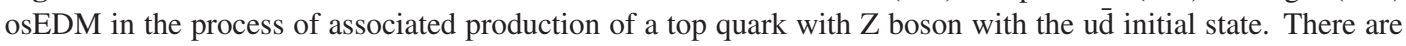
plots for $\mathrm{Z}$ boson (top left), top quark (top right) and bottom quark (bottom left), and invariant mass for $\mathrm{Z}$ and $\mathrm{b}$ (bottom right). The distributions of each coupling are normalized to the corresponding cross sections.

contribution from such anomalous couplings. Necessary calculations of these processes have been performed. The difference in the shape of the kinematic distributions owing to the contribution of the osEDM couplings are demonstrated in this article. The demonstrated difference in the kinematic distributions is promising for the search and achieving the first experimental limits on these couplings. More accurate estimation of the sensitivity to osEDM couplings requires simulation of the hadronization with detector response and careful estimation of the background contributions. 

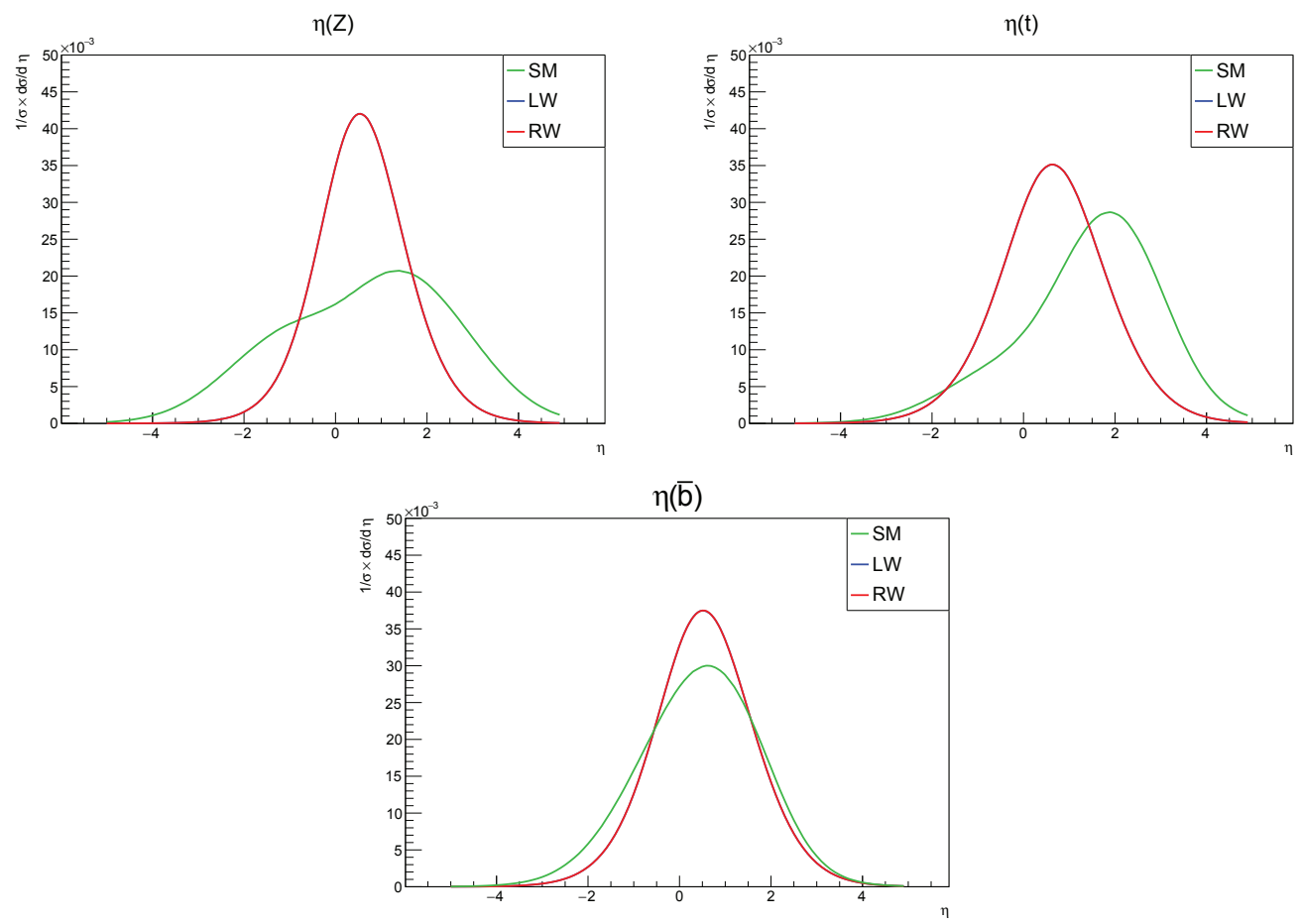

Figure 4. Pseudorapidity distributions for the SM interaction (SM) and pure left (LW) and right (RW) osEDM in the process of associated production of a top quark with $\mathrm{Z}$ boson with the ud initial state. There are plots for $\mathrm{Z}$ boson (top left), top quark (top right) and bottom quark (bottom). The distributions of each coupling are normalized to the corresponding cross sections.
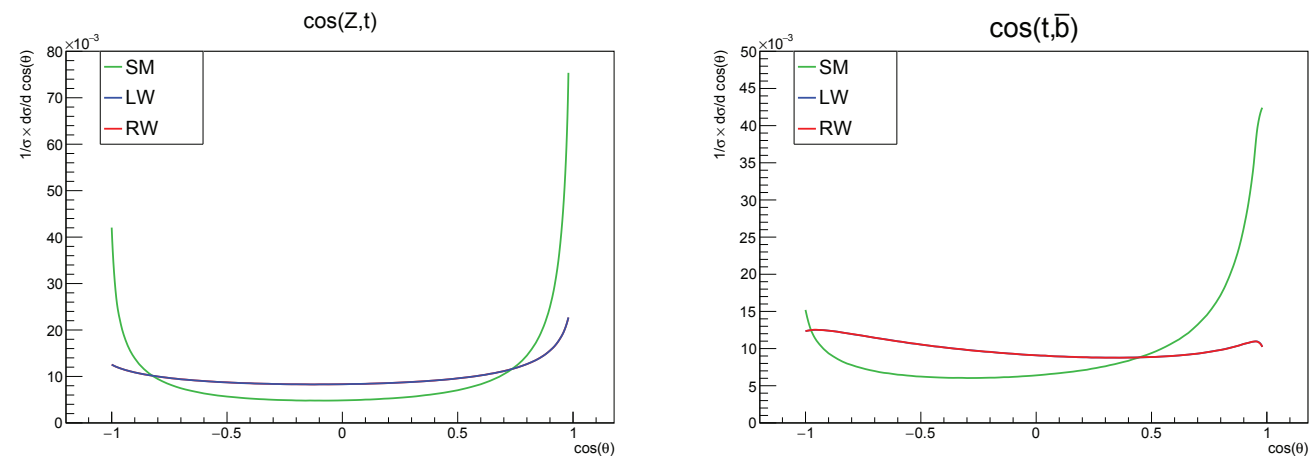

Figure 5. Angular distributions for the SM interaction (SM) and pure left (LW) and right (RW) osEDM in the process of associated production of a top quark with $\mathrm{Z}$ boson with the ud initial state. There are plots for cosine of angle between $\mathrm{Z}$ boson and top quark (left), and cosine of angle between top and bottom quarks (right). The distributions of each coupling are normalized to the corresponding cross sections. 

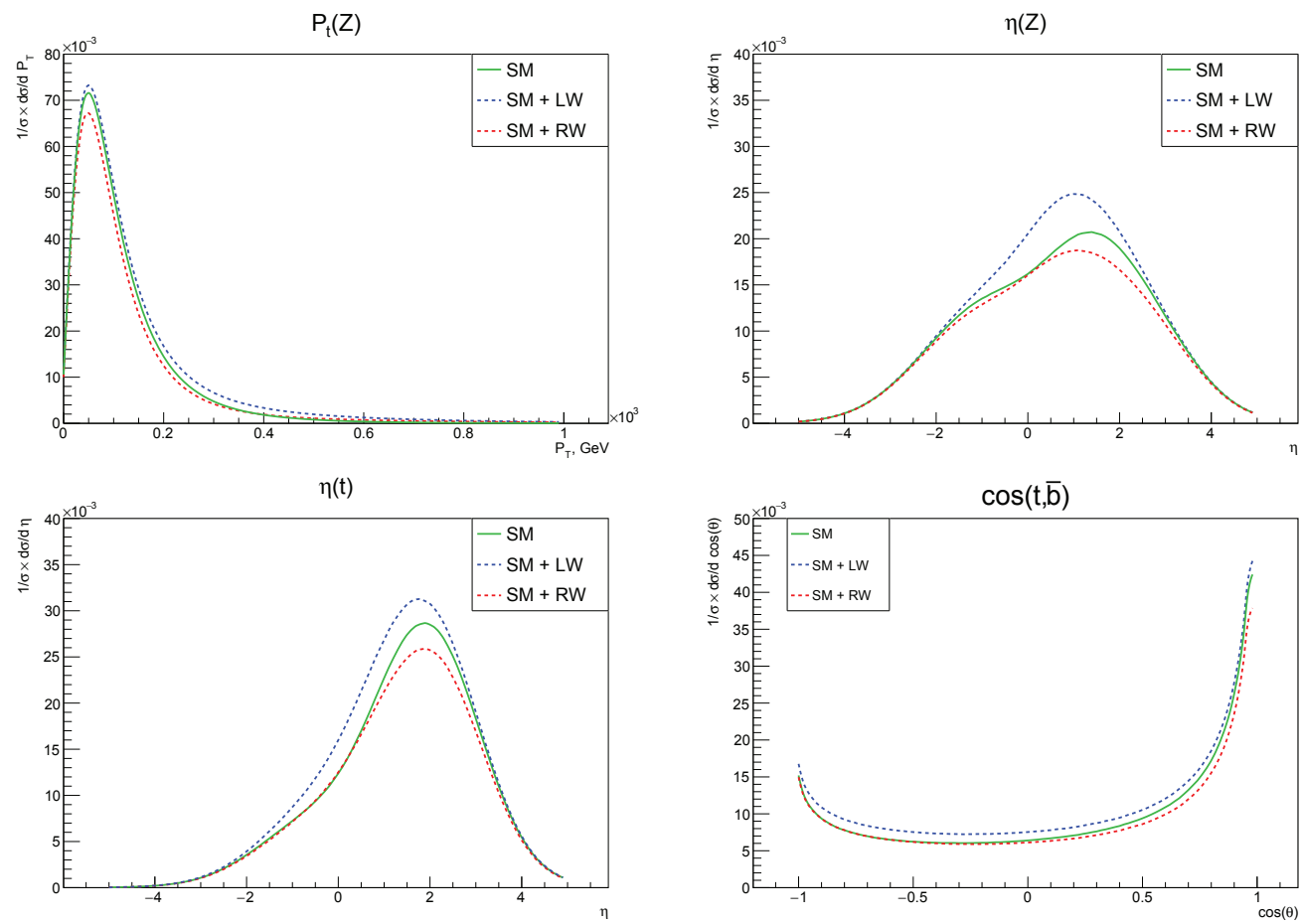

Figure 6. Distributions for the SM interaction (SM), SM and left osEDM (SM+LW), and SM and right anomalous coupling $(\mathrm{SM}+\mathrm{RW})$, including interference, in the process of associated production of a top quark with $\mathrm{Z}$

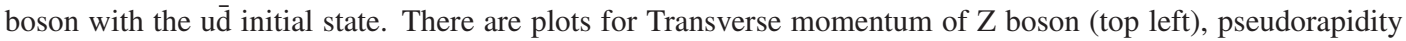
of $\mathrm{Z}$ boson (top right) and top quark (bottom left), and cosine of angle between top and bottom quarks (bottom right). The all distributions are normalized to the cross section of SM interaction.
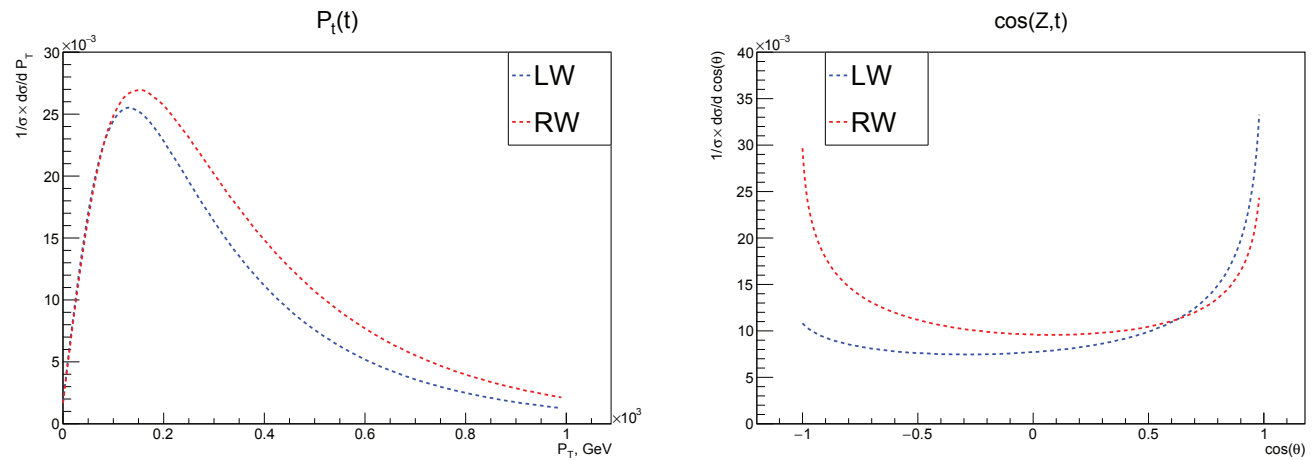

Figure 7. Distributions for the pure left (LW) and right (RW) anomalous couplings, in the process of associated production of a top quark with $\mathrm{Z}$ boson with the $\mathrm{cs}$ initial state. There are plots for Transverse momentum of top quark (left), and cosine of angle between $\mathrm{Z}$ boson and t quark (right). The all distributions are normalized to the cross section of left osEDM. 


\section{Acknowledgements}

The work was supported by grant 16-12-10280 of Russian Science Foundation.

\section{References}

[1] G. L. Kane, G. A. Ladinsky and C. P. Yuan, Phys. Rev. D 45 (1992) 124.

[2] W. Buchmuller and D. Wyler, Nucl. Phys. B 268 (1986) 621.

[3] K. Whisnant, J. M. Yang, B. L. Young and X. Zhang, Phys. Rev. D 56 (1997) 467

[4] E. Boos, M. Dubinin, M. Sachwitz and H. J. Schreiber, Eur. Phys. J. C 16 (2000) 269

[5] J. A. Aguilar-Saavedra, J. Carvalho, N. F. Castro, F. Veloso and A. Onofre, Eur. Phys. J. C 50 (2007) 519

[6] V. M. Abazov et al. [D0 Collaboration], Phys. Lett. B 708 (2012) 21

[7] V. Khachatryan et al. [CMS Collaboration], JHEP 1501 (2015) 053

[8] V. Khachatryan et al. [CMS Collaboration], JHEP 1702 (2017) 028

[9] F. Larios and C. P. Yuan, Phys. Rev. D 55 (1997) 7218

[10] E. Boos et al. [CompHEP Collaboration], Nucl. Instrum. Meth. A 534 (2004) 250

[11] A. Semenov, Comput. Phys. Commun. 201 (2016) 167 